

\title{
Topical gabapentin gel alleviates allodynia and hyperalgesia in the chronic sciatic nerve constriction injury neuropathic pain model.
}

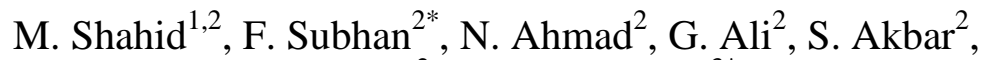 \\ K. Fawad ${ }^{2}$, R. D. E. Sewell $3^{3 *}$
}

${ }^{1}$ Department of Pharmacy, Sarhad University of Science and Information Technology, Peshawar, Pakistan
${ }^{2}$ Department of Pharmacy, University of Peshawar 25120, Peshawar, Pakistan
${ }^{3}$ Cardiff School of Pharmacy and Pharmaceutical Sciences, Cardiff University, Cardiff CF10 3NU, UK.

\section{Original Article}

Running head: Topical gabapentin and allodynia

\section{Conflict of interest}

None declared

\section{*Correspondence:}

Professor Fazal Subhan

Email: fazal_subhan@upesh.edu.pk

Professor Robert D. E. Sewell

Email: sewell@ cardiff.ac.uk 


\section{Abstract:}

Background: Systemic gabapentin is a mainstay treatment for neuropathic pain though there are side effects. Localized therapy may curtail such side effects so a topical gabapentin dermal application was examined in the chronic constriction injury (CCI) model of neuropathic pain.

Methods: Partial denervation CCI was achieved by rat sciatic nerve ligation. Gabapentin gel $(10 \% \mathrm{w} / \mathrm{w})$ was applied three-times daily on the ipsilateral or contralateral plantar surface of the hind-paw while in a concurrent systemic study, gabapentin was intraperitoneally administered daily $(75 \mathrm{mg} / \mathrm{kg}$ ) for 30 days. Tests for static- and dynamic- mechano-allodynia (paw withdrawal threshold [PWT] to von-Frey filament application and latency [PWL] to light brushing), coldallodynia (paw withdrawal duration [PWD] to acetone), heat- (PWL and PWD) and mechanohyperalgesia (PWD to pin-prick) were utilized to assess pain while effects on locomotion (open field) and motor balance (rotarod and footprint-analysis) were measured on days 5-30 postsurgery.

Results: Topical application of gabapentin gel ipsilaterally but not contralaterally alleviated CCI-induced static- (days 10-30) and dynamic-allodynia (days 15-30), suppressed cold-allodynia (days 10-30), heat- (days 15-30) and mechano-hyperalgesia (days 5-30) indicating a local action. Systemic gabapentin exhibited similar pain profiles but was associated with motor impairment. The gabapentin gel formulation afforded desirable neuropathic pain alleviating effects devoid of unwanted systemic side-effects.

Conclusions: These outcomes disclose an expedient pharmacological validation of the effectiveness of topical gabapentin gel against an extensive range of nociceptive stimulus modalities utilizing the CCI-induced neuropathic pain model. They also advocate further clinical studies on topical gabapentin with regard to certain neuropathic pain syndromes.

\section{Keywords:}

Gabapentin gel; neuropathic pain; allodynia and hyperalgesia; localized therapy, no motor impairment 


\section{Introduction:}

Neuropathic pain is a chronic condition caused by a lesion or disease of the central or peripheral somatosensory nervous system (IASP, 1994). The condition may also develop secondary to other pathological disorders such as diabetes mellitus, cancer, infections, autoimmune diseases and HIV infection (Campbell and Meyer, 2006; Costigan et al., 2008). Neuropathic pain is characterized by development of allodynia and hyperalgesia (IASP, 1994) experienced either simultaneously or independently (Jensen and Finnerup, 2014).

Management of peripheral neuropathic pain is guided by two principles: treatment of the underlying condition and strategies designed to relieve peripheral nerve pain irrespective of cause. Systemic pharmacotherapy is the mainstay treatment and NICE guidelines suggest offering a choice of amitriptyline, duloxetine, gabapentin or pregabalin (gabapentinoids) (Bouhassira et al., 2014; Marchettini et al., 2016; Moulin et al., 2014) as initial treatment for all neuropathic pain (except trigeminal neuralgia), tramadol being considered only if rescue therapy is needed (NICE, 2013). Unfortunately, the use of these agents can be limited by the extent of pain relief provided and the occurrence of side effects (Attal, 2001; Harden, 1999; Mendell and Sahenk, 2003; Moulin et al., 2007).

Gabapentin was first approved by the MHRA in 1993 as an oral add-on therapy for treatment of partial seizures and later in 2000 for peripheral neuropathy. The efficacy of gabapentin has been demonstrated in clinical trials and in several preclinical models of neuropathic pain (Field et al., 1997; Rowbotham et al., 1998). Lamentably, the efficacy of systemic gabapentin in patients 
suffering from neuropathic pain may be attended by side effects such as dizziness, somnolence, ataxia, lethargy, and convulsions (Rose and Kam, 2002).

Given that a delay in treating a painful peripheral area can only lead to ongoing worsening pain, topical agents may provide a valuable first line treatment ensuring pain reduction with minimal central side effects. Evidence suggests that localized cutaneous neuropathic pain can be treated effectively and safely by topical medications when oral therapies have failed or have been stopped due to side effects. Topical analgesics applied locally to the skin or to specialized compartments modify pain by actions on sensory nerve endings and/or adjacent cellular elements. Employing this approach, there are low systemic drug levels, good tolerability, few drug interactions and combination with oral formulations is feasible (Sawynok, 2014). However, only a few topical medications are licensed for neuropathic pain (Zur, 2014). In fact, the only topical agent recommended by NICE is capsaicin cream for patients with localized neuropathic pain who wish to avoid, or cannot tolerate, oral treatments (NICE, 2013). The side effects of systemic gabapentin treatment may be diminished by localized therapy (Hiom et al., 2015a). Moreover, a topical formulation of gabapentin $\left(\mathrm{Gaba}-\mathrm{Gel}^{\mathrm{TM}}\right)$, when applied to an affected site has been shown to relieve postherpetic neuralgia and potentially other painful neuropathic syndromes (Hiom et al., 2015b). In another investigation, topical 10\% gabapentin gel alleviated both mechanical allodynia and vulvodynia in a novel animal model of streptozotocin induced diabetic neuropathic nociception (Ali et al., 2015).

In the present study, we evaluated this topical formulation using a more widely used rat model of chronic sciatic nerve constriction induced neuropathic pain (Bennett and Xie, 1988) with the aim of establishing additional validity of the effectiveness of topical gabapentin against neuropathic nociception. Thus, gabapentin gel was applied for 30 days and nociception was assessed in the 
model using a number of behavioral testing paradigms for allodynia and hyperalgesia. In addition, tests for locomotor activity and motor coordination were carried out to evaluate the effect of gabapentin gel formulation on neuronal function in comparison to systemic gabapentin.

\section{Methods:}

\section{Chemicals:}

Gabapentin gel 10\% w/w and the control gel base (an oil in water gel comprising xanthan gum hydrocolloid with polyacrylamide minus the active pharmaceutical ingredient) were supplied by St Mary's Pharmaceutical Unit (SMPU, Cardiff, UK) under their Manufacturer's Special License (MS). Gabapentin for systemic administration was obtained from Lowitt Pharmaceuticals, Pakistan, and the following were used: xylazine (Xylaz ${ }^{\circledR}, 20 \mathrm{mg} / \mathrm{ml}$, France) and ketamine (Ketarol®, 50 mg/ml, Global Pharmaceutical, Pakistan).

\section{Animals:}

Male Sprague Dawley rats, weighing 300-450 gm and maintained in a $12 \mathrm{~h} / 12 \mathrm{~h}$ light/dark cycle at $22 \pm 2{ }^{\circ} \mathrm{C}$ were used in the experiments. The animals were allowed free access to food and water. All the experimental procedures on animals were performed in compliance with the UK Animals (Scientific Procedures) Act 1986 and according to the rules and ethics set forth by the ethical committee of the Department of Pharmacy, University of Peshawar. Approval for the study was granted with the registration number: 13/EC-15/Pharm.

\section{Induction of the neuropathic pain model:}

A neuropathic pain model involving chronic constriction injury (CCI) producing partial denervation of the sciatic nerve was employed (Bennett and Xie, 1988). Briefly, each rat was 
anesthetized with an intraperitoneal injection of a mixture of xylazine $(10 \mathrm{mg} / \mathrm{kg})$ and ketamine $(100 \mathrm{mg} / \mathrm{kg})$ and then placed in a prone position on a heat regulated pad with an application of lubricating ophthalmic ointment (Lacri-Lube ${ }^{\circledR}$, Barrett Hodgson, Pakistan) to the eyes. The left thigh was elevated and the fur on the posterior skin was shaved using electric clippers (Ding Ling RF-602, China). The exposed skin was swabbed with a povidone iodine topical $10 \% \mathrm{w} / \mathrm{v}$ solution (Pyodine ${ }^{\circledR}$, Brookes Pharma, Pakistan). A 3-4 cm incision was made parallel to the long axis of the femur and down the center of the biceps femoris muscle. Under a magnifying glass, the connective tissue between the gluteus superficialis and the biceps femoris muscles was cut. Using ribbon retractors, the gap between the two muscles was widened and approximately 10 $\mathrm{mm}$ of the sciatic nerve (proximal to the sciatic trifurcation) was freed from the surrounding connective tissue with a micro-scissor. Four ligatures (Chromic catgut suture 4/0, metric 2, Ethicon, Pakistan) were tied with a double knot, $1 \mathrm{~mm}$ apart and proximal to the trifurcation of the sciatic nerve. The constriction of the nerve was minimal and was immediately stopped when a brief twitch was observed. The muscle layer was then closed with sutures (Silk braided 2/0, metric 2, Zhejiang Medicines \& Health Products, China) and the skin was fastened using a surgical stapler (Manipler AZ-35W, Japan). In sham operated rats, an identical operation was performed, except that the sciatic nerve was not ligated (Austin et al., 2012).

\section{Treatment groups:}

\section{Experiment 1 (ipsilateral paw application):}

To assess the effect of topical gabapentin, a uniform quantity of $10 \%$ gabapentin gel (1.0 $\left.\mathrm{mL} / \mathrm{cm}^{2}\right)$ was applied topically three times daily $(08.00,12.00$ and $16.00 \mathrm{~h})$ on the ipsilateral plantar surface of the operated left hind paw. Similarly, the control animals received an 
equivalent application of control gel $\left(1.0 \mathrm{~mL} / \mathrm{cm}^{2}\right)$ on the operated left hind paw. In the case of the systemic study, gabapentin was administered once daily at a dose of $75 \mathrm{mg} / \mathrm{kg}$, i.p (Ali et al., 2015). The animals were randomly assigned to the following treatment groups $(n=6)$ and the study was continued for 30 days during which the operated left paw was tested:

Group 1: sham operated control.

Group 2: chronic constriction injury (CCI) control.

Group 3: CCI + GG (gabapentin gel on the ipsilateral paw).

Group 4: CCI + CG (control gel on the ipsilateral paw).

Group 5: CCI + gabapentin (75 mg/kg, i.p).

Group 6: sham operated animals + GG on the ipsilateral paw.

Group 7: sham operated animals + CG on the ipsilateral paw.

Group 8: sham operated animals + gabapentin (75 mg/kg, i.p).

Experiment 2 (contralateral paw application):

To assess if the gabapentin content in the gel gained access to the systemic circulation through local application, a uniform quantity of $10 \%$ gabapentin gel or control gel $\left(1.0 \mathrm{~mL} / \mathrm{cm}^{2}\right)$ was applied topically three times daily $(08.00,12.00$ and $16.00 \mathrm{~h})$ on the plantar surface of the contralateral right hind paw (opposite to the operated left hind paw). In addition to groups in experiment 1 , the animals were randomly divided into the following groups $(n=6)$ and the topical treatment was continued for 30 days during which the operated left paw was tested:

Group 9: CCI + GG (gabapentin gel on the contralateral paw).

Group 10: CCI + CG (control gel on the contralateral paw).

Group 11: sham operated animals + GG on the contralateral paw.

Group 12: sham operated animals + CG on the contralateral paw. 


\section{Pain assessment:}

The animals were transferred to a specially designed elevated wire mesh bottom table and were acclimatized for 15-45 min before behavioral assessment. Behavioral tests for static and dynamic allodynia, heat hyperalgesia, mechanical hyperalgesia and cold allodynia were measured 5 days before-surgery and on days $5,10,15,20,25$ and 30 post-surgery.

\section{Static mechano-allodynia:}

A series of 8 von Frey filaments $(0.4,0.70,1.20,2.00,3.63,5.50,8.50$, and $15.10 \mathrm{~g})$ (Stoelting USA) were applied perpendicularly to the mid-plantar surface of the operated left hind paw to an extent that caused the hairs to bend (Chaplan et al., 1994). Each von Frey filament was applied for a period of up to $6 \mathrm{~s}$ as a cut-off time or until a positive response occurred. Lifting of the paw or flinching immediately upon removal of the filament was recorded as a positive response and a succeeding von Frey filament of lower force was applied for the following recording. In the case of an absence of response, the subsequent von Frey filament of higher force was applied. This procedure was continued until four measurements were taken after the first change in direction (positive response) or five consecutive negative responses $(2.00,3.63,5.50,8.50$, and 15.10-g

force) or four consecutive positive responses $(2.00,1.20,0.70,0.4-\mathrm{g}$ force). A force of $15.10 \mathrm{~g}$ was selected as the cut-off force at which point further application ceased. von Frey filaments were applied at intervals of several seconds in order to avoid any influence of previous stimuli on behavior. Any ambulation was noted as an indefinite response, and the stimulus was repeated. The pattern of response was converted to the 50\% withdrawal threshold (PWT, g) using an Excel sheet specified by Mao-Ying et al (Mao-Ying et al., 2014) and based on a previously described method (Chaplan et al., 1994). 


\section{Dynamic mechano-allodynia:}

Dynamic allodynia was assessed by lightly stroking the mid-plantar surface of the operated left hind paw with a cotton bud. Lifting or licking the paw was considered as a withdrawal response and the time taken to show a withdrawal reaction was considered as the paw withdrawal latency (PWL). Acut off time of $15 \mathrm{~s}$ was imposed (Nakazato-Imasato et al., 2009).

\section{Heat hyperalgesia:}

The mid-plantar surface of the operated left hind paw was lightly touched with a heated plate maintained at a constant temperature $\left(56^{\circ} \mathrm{C}\right)$. The heat source was adjusted at the beginning of the experiment to yield a paw flick within $\sim 10 \mathrm{~s}$. The paw withdrawal latency (PWL) and duration (PWD) were recorded with a minimal value of $0.5 \mathrm{~s}$ and a maximum of $10 \mathrm{~s}$ (Bennett et al., 2003).

\section{Mechano-hyperalgesia:}

Animals were placed on an elevated grid and the tip of an ordinary safety pin was pressed against the skin of the mid-plantar surface of the operated left hind-paw such that the skin was dimpled but not penetrated. The duration of paw withdrawal (PWD) was recorded, with an arbitrary minimal time of $0.5 \mathrm{~s}$ (for the brief normal response) and a maximal cut-off of $15 \mathrm{~s}$ (Decosterd and Woolf, 2000).

\section{Cold allodynia:}

A $50 \mu \mathrm{L}$ volume of acetone was delicately sprayed onto the mid-plantar surface of the operated left hind paw, using a blunt needle connected to a syringe without touching the skin. The 
duration of the withdrawal response (PWD) was recorded with an arbitrary minimal value of 0.5 s and a maximum of $15 \mathrm{~s}$ (Decosterd and Woolf, 2000).

\section{Locomotor activity:}

General locomotor activity was measured in an open field paradigm consisting of a behavioral arena with a floor area of $50 \times 40 \mathrm{~cm}$ and divided into four equal quadrants by lines (Subhan et al., 2010). On the test day, each animal was habituated to the test apparatus for $30 \mathrm{~min}$. The number of lines crossed by each animal was recorded for $20 \mathrm{~min}, 1 \mathrm{~h}$ post topical or systemic gabapentin treatment. Locomotor behavior was subsequently observed post surgically on days 5 , $10,15,20,25$ and 30.

\section{Motor coordination:}

\section{Rota rod:}

A purpose built accelerating rotarod was used in which the animals were placed on a rotating drum with the speed increasing from 4 to $40 \mathrm{rpm}$ over $5 \mathrm{~min}$, forcing them to walk forward to avoid falling off (Chen et al., 2014). The latency to falling off the rotarod was measured and each animal was given three trials on the test day before drug treatment. The effect on motor performance was then evaluated on days $5,10,15,20,25$ and 30 after surgery, $1 \mathrm{~h}$ post topical application or systemic administration of gabapentin.

\section{Footprint analysis:}

Gait analysis was performed using footprint patterns (walking tracks) (Carter et al., 2001). The fore and hind paws of each animal were coated with two different colored nontoxic inks and they were allowed to walk along a narrow, paper covered corridor, leaving a track of footprints. The 
footprints were allowed to dry and the patterns of the un-operated right side were measured for stride length (average distance of forward movement between each stride) and overlap between forepaw and hindpaw placement (distance between the front and hind footprints on each side). On the test day, each animal was given two trials before treatment. The effect was evaluated $1 \mathrm{~h}$ after topical application or systemic administration of gabapentin post-surgery on days $5,10,15$, 20,25 and 30.

\section{Statistical analysis:}

Animals were randomly assigned to each treatment group. Results are presented as mean \pm SEM. The datawere analyzed using two-way repeated measures analysis of variance (ANOVA) (time $\times$ treatment) followed by post hoc Bonferroni test. A value of $P \leq 0.05$ was accepted as significant.

\section{Results:}

\section{Effect of topical ipsilateral gabapentin gel on neuropathic allodynia and hyperalgesia}

The CCI operated control animals developed significant hypersensitivity to innocuous mechanical von Frey filament stimulation i.e. decreased PWT (static mechano-allodynia), (days 5-30, $P<0.001$, Fig 1A) and to light brushing (dynamic mechano-allodynia), evidenced by diminished PWL (days 10-30, $P<0.01$ and $P<0.001$, Fig 1B) as compared to the sham operated animals. These animals displayed a significant decrease $(P<0.001)$ in the latency (Fig 1C) and an increase $(P<0.001)$ in the duration of withdrawal response to the heat stimulus (days 5-30, Fig 1D). Additionally, the duration of flexion withdrawal evoked by a pin prick stimulus (mechanical hyperalgesia, Fig 2A) or a drop of acetone (cold allodynia, Fig 2B) was significantly increased $(P<0.001), 5$ days after surgery and this was maintained for the subsequent study duration (30 days) when compared to the sham operated control animals. 
Ipsilateral topical and systemic gabapentin produced significant main dose effects in the CCI induced static mechano-allodynia $[F(7,210)=89.88, P<0.0001]$ (Fig 1A), dynamic mechanoallodynia $[F(7,245)=25.05, P<0.0001]($ Fig $1 \mathrm{~B})$, heat hyperalgesia including PWL $[F(7,245)$ $=93.46, P<0.0001]$ Fig $1 \mathrm{C})$ and PWD $[F(7,245)=67.05, P<0.0001]($ Fig $1 \mathrm{D})$, mechanical hyperalgesia $[F(7,245)=114.57, P<0.0001](F i g 2 A)$ and cold allodynia $[F(7,245)=200.81$, $P<0.0001$ ] (Fig 2B). Application of topical 10\% gabapentin gel three times daily to the ipsilateral plantar surface of the operated left hind-paw alleviated the CCI induced neuropathic pain as evidenced by significantly increased PWT (day 10-30, $P<0.01$ and $P<0.001$ ) (Fig 1A), PWL to light brushing (day 15-30, $P<0.05, P<0.01$ and $P<0.001$ ) (Fig 1B), increased PWL (day 10-30, $P<0.05, P<0.01$ and $P<0.001$ ) (Fig 1C) and shortened PWD (day 20-30, $P<$ $0.05, P<0.01$ and $P<0.001$ ) to heat stimulus (Fig 1D), reduced PWD to pin prick (day 5-30, $P$ $<0.05$ and $P<0.001$ ) (Fig 2A) and a drop of acetone (day 5-30, $P<0.01$ and $P<0.001$ ) (Fig 2B). Similarly, animals treated once daily with systemic gabapentin $(75 \mathrm{mg} / \mathrm{kg})$ significantly diminished neuropathic allodynia (day 5-30, $P<0.05$ and $P<0.001$ ) and hyperalgesia (day 5-30, $P<0.05, P<0.01$ and $P<0.001)$ Fig 1 and Fig 2$)$.

The control gel treated animals were not found to express modified allodynia and hyperalgesia when compared to the CCI operated untreated animals. Moreover, no aberrant behavioral changes were detected in the sham operated control or sham plus gabapentin gel/control gel/systemic gabapentin treated animals at any time during the 30 days of observation.

\section{Effect of topical contralateral gabapentin gel on neuropathic allodynia and hyperalgesia}

The CCI procedure produced a significant decrease in the nociceptive threshold to the innocuous mechanical von Frey filament (day 5-30, $P<0.001$ ) (Fig 3A) and reduced the withdrawal 
latency upon lightly stroking with a cotton bud (day 10-30, $P<0.001$ ) (Fig 3B). It decreased the latency (Fig 3C) and escalated the withdrawal response (Fig 3D) to normally non-painful heat stimuli (day $5-30, P<0.001$ ). It also exaggerated the withdrawal response to a noxious stimuli evoked pain and hastened the flexion reflex to a cold stimulus (day 5-30, $P<0.001$ ) (Fig 4A and 4B). Conversely, treatment with topical or systemic gabapentin diminished the CCI induced changes in the behavioral paradigms of static allodynia $[F(11,385)=194.75, P<0.0001]$ (Fig 3A), dynamic allodynia $[F(11,385)=61.21, P<0.0001]$ (Fig 3B), thermal hyperalgesia including PWL $[F(11,385)=115.34, P<0.0001](F i g 3 C)$ and PWD $[F(11,385)=279.55, P<$ 0.0001] (Fig 3D), punctate hyperalgesia $[F(11,385)=250.79, P<0.0001]$ (Fig 4A) and cold allodynia $[F(11,385)=414.55, P<0.0001]$ (Fig 4B). Thus, topical application of $10 \%$ gabapentin gel three times daily on the ipsilateral operated left hind-paw significantly alleviated the diminished PWT (day 5-30, $P<0.001$ ) (Fig 3A), increased the PWL to non-painful lightpressure dynamic stimuli (day 15-30, $P<0.001$ ) (Fig 3B), reduced painful temperature sensation (elevated the PWL, reduced PWD) (day 5-30, $P<0.01$ and $P<0.001$ ) (Figs 3C and 3D), lessened the PWD to a noxious stimuli evoked pain and non-painful cold stimuli (days 5-30, $P<$ 0.001) (Fig 4A).However, when this gel formulation was applied to the contralateral hindpaw in the CCI operated animals, it failed $(P>0.05)$ (Fig 4B) to relieve the deviant responses of the neuropathic pain conditions. On the other hand, utilizing the systemic route, daily intraperitoneal administration of gabapentin $(75 \mathrm{mg} / \mathrm{kg})$ afforded a robust static, dynamic and cold antiallodynic (day 5-30, $P<0.001$ ), thermal and mechanical anti-hyperalgesic effects (day 5-30, $P<$ 0.01 and $P<0.001$ ) (Figs 3 and 4 ).

Likewise, topical application of control gel on the ipsilateral or contralateral hind paw was unable $(P>0.05)$ to achieve any therapeutic improvement in the evoked sensations delineating 
the neuropathic pain. The sham operated control as well as sham operated plus gabapentin gel/control gel on either the ipsilateral or contralateral side or systemic gabapentin treated animals did not exhibit any hypersensitivity during the entire study duration.

\section{Effect of topical gabapentin gel on locomotor performance}

Unilateral ligation of the sciatic nerve decreased the overall locomotor performance causing a significant reduction in the total number of lines crossed by the CCI operated animals compared to the sham operated controls from days 10-30 of the study. ANOVA analysis revealed a significant main effect of time $[F(6,385)=11.82, P<0.0001]$ and treatment $[F(11,385)=$ 26.78, $P<0.0001]$. However, no significant time $\times$ treatment interaction was observed for line crosses $[F(66,385)=1.22, P<0.1267]$. Subsequent post hoc analyses revealed that the CCI plus systemic gabapentin treated animals displayed a significant increase in the total number of lines crossed in the behavioral arena compared to the CCI untreated control animals. The increase in locomotion remained significant between day $10(P<0.01)$ and day $30(P<0.001)$ (Fig 5). Similarly, the sham operated plus systemic gabapentin treated animals also exhibited a significant increase in line crosses when assessed on day $5(P<0.05)$ to day $30(P<0.001)$. No significant effect was observed for the control or gabapentin gel on either ipsilateral or contralateral hindpaw except on day 20, where the CCI plus ipsilateral gabapentin gel produced a significant increase $(P<0.01)$ in locomotor activity as compared to the CCI untreated animals (Fig 5). The sham operated plus ipsilateral or contralateral control gel/gabapentin gel significantly increased $(P<0.05, P<0.01)$ the number of times the animal physically line crossed when compared to the untreated CCI operated animals from day 5 of surgery to the end of study duration (day 30). 


\section{Effect of topical gabapentin gel on motor co-ordination and balance}

The ability of an animal to poise on a rotating drum was assessed to measure the effect of the gel formulation and systemic gabapentin on motor coordination and balance. The analysis revealed a significant main effect of time $[F(6,385)=49.54, P<0.0001]$ and treatment $[F(11,385)=$ 92.65, $P<0.0001]$. In addition, there was significant time $\times$ treatment interaction for latency to fall off the rotating drum $[F(66,385)=3.93, P<0.0001]$. The CCI procedure caused a deficit in motor function as the fall-off time was significantly decreased post-surgery from day 5 to day 30 $(P<0.01, P<0.001)$ (Fig 6). Systemic gabapentin was associated with significant motor incoordination as indicated by a profound decrease $(P<0.001)$ in the latency time observed with the CCI or sham operated- plus systemic gabapentin-treated animals as compared to sham operated untreated controls throughout the study duration. Moreover, the ipsilateral or contralateral application of gabapentin gel also produced a variable effect on muscular balance but of less severity $(P<0.05, P<0.01)$ as compared to the systemic gabapentin treatment (Fig 6). The sham operated controls as well as those ipsilaterally or contralaterally treated alone with control gel/gabapentin gel animals did not display any motor incoordination as no significant change in the rotarod latency was produced during any time in the entire study duration.

The effect of gel and systemic gabapentin on motor balance was further corroborated by gait analysis using footprint patterns (walking tracks). Hence, neither the CCI procedure, nor the treatments had any effect on the stride length as no significant difference was observed in the forward movement distance during each stride $[$ time $=[F(6,385)=1.14, P<0.3583]$, treatment $=[F(11,385)=2.02, P<0.0254]$, interaction $[F(66,385)=0.94, P<0.6027]$. However, a significant change was observed in the overlap between the forepaw and hindpaw placement [time $=(F(6,385)=5.66, P=0.0003)$, treatment $=(F(11,385)=50.69, P<0.0001)$, interaction 
$=(F(66,385)=2.32, P<0.0001)]($ Fig7). The CCI animals administered daily with systemic gabapentin $(75 \mathrm{mg} / \mathrm{kg}$, i.p) produced significant foot displacement $(P<0.001)$ during the entire study duration as compared to the sham operated control animals (Fig 7). Likewise, the sham operated plus systemic gabapentin treated animals also showed a significant deficit $(P<0.001)$ in the uniformity of step alternation. The sham operated or the CCI operated plus control gel/gabapentin gel groups as well as those treated alone with control gel/gabapentin gel displayed no effect $(P>0.05)$ on the distance between the center of the footprints on the same side throughout the study period.

\section{Discussion:}

In the present study, we demonstrated that $10 \% \mathrm{w} / \mathrm{w}$ topical gabapentin gel applied three times daily produced significant anti-allodynic and anti-hyperalgesic effects in a chronic sciatic nerve constriction injury model in the rat. The chronic constriction injury model has been shown to be sensitive to a number of agents used clinically for the symptomatic treatment of chronic neuropathic pain. Hence, a series of compounds has been used to modulate neuropathic allodynia and other manifestations of neuropathic pain (De Vry et al., 2004). In this context, systemic gabapentin appears to be an effective treatment for neuropathic pain, because of its unique effect

on voltage dependent calcium ion channels located postsynaptically in the spinal dorsal horn. Consequently, it may interrupt the series of events that probably lead to the experience of neuropathic sensation (Rose and Kam, 2002). Different neuropathic pain conditions including complex regional pain syndromes, deafferentation neuropathies, central post stroke pain and pain associated with multiple sclerosis have shown positive responses to therapy with oral gabapentin. In this connection, randomized placebo controlled trials have demonstrated its effectiveness in post herpetic neuralgia, diabetic neuropathy, mixed neuropathic pain and cancer neuropathic pain 
(Bennett and Simpson, 2004). In laboratory animals, gabapentin can inhibit neuropathic pain behaviors induced by peripheral nerve injury. Accordingly, systemic gabapentin has been shown to partially suppress mechanical allodynia and reverse heat hyperalgesia, mechanical hyperalgesia and cold allodynia (Gilron and Flatters, 2006). Similarly, in our present study, gabapentin showed pronounced anti-hyperalgesic and anti-allodynic properties after daily intraperitoneal administration during the 30 day experimental study and this further validates its utility as a treatment for neuropathic pain.

Topical drugs have multiple advantages because they act locally on damaged or dysfunctional soft tissues or peripheral nerves so systemic exposure is minimized as are systemic adverse effects and drug-drug interactions. Furthermore, topical administration conceivably prevents the need to titrate doses to tolerable levels. The importance of the skin as a target for topical and systemic medication to treat neuropathic pain is further supported by studies demonstrating the importance of the skin as a neuroimmunocutaneous system. Correspondingly, there are signals between neural and non-neural skin cells, and a large variety of receptors and endogenous ligands located on peripheral nociceptors and non-neural cells (Stanos, 2007; Zur, 2014). In the current study, topical application of gabapentin allayed neuropathic-like pain conditions and provided effectiveness similar to systemic gabapentin. Moreover, gabapentin gel application to the CCI contralateral paw proved ineffective against neuropathic-like pain parameters. Consequently, it might be reasonably concluded that the observed activity on the ipsilateral paw was truly local and did not stem from systemic absorption. Furthermore, the topical gabapentin gel formulation was devoid of any observed side effects commonly encountered with the clinical systemic use of gabapentin such as sedation, dizziness, somnolence, asthenia, ataxia, amblyopia, headache and nausea (McLean et al., 1999). This has been confirmed by using different testing 
paradigms in which gabapentin by the systemic (intraperitoneal) route produced only a transient increase in the locomotor behavior but induced considerable impairment in the motor coordination. These unwanted effects have been ostensibly prevented by the localized gel formulation of gabapentin, yet retaining the beneficial effects desirable in the neuropathic pain management. The efficacy and safety of topical medications for the treatment of neuropathic pain has been demonstrated and several topical medications have been investigated in well-designed trials. These include a 5\% lidocaine medicated plaster, a capsaicin $8 \%$ patch, topical clonidine gel $(0.1 \%)$, combination of doxepin hydrochloride $(3.3 \%)$ and capsaicin $(0.025 \%)$, topical baclofen $0.76 \%$, amitriptyline $3 \%$, and ketamine $1.5 \%$ in pluronic-lecithin organogel, $2 \%-6 \%$ gabapentin in lipoderm base, amitriptyline $2 \%$, baclofen $2 \%$ in lipoderm cream base, and aspirin 2.5\%-7.5\% dissolved/suspended in diethyl ether (Zur, 2014).

In our previous study involving diabetes induced neuropathy, topical application of gabapentin $10 \% \mathrm{w} / \mathrm{w}$ gel applied three times daily significantly ameliorated both static and dynamic allodynia as well as static and dynamic vulvodynia induced by a single injection of streptozotocin in female rats (Ali et al., 2015). This same formulation when applied as $6 \% \mathrm{w} / \mathrm{w}$ gabapentin three times per day to the affected site also alleviated postherpetic neuralgia and other neuropathic pain syndromes including postsurgical pain, complex regional pain syndrome, painful diabetic polyneuropathy, vulvovaginodynia, trigeminal neuralgia, autonomic cephalalgia, pudendal neuralgia and coccygodynia in patients (Hiom et al., 2015b). The results from these studies are well corroborated with our current findings in a chronic sciatic nerve constriction injury model where the application of $10 \% \mathrm{w} / \mathrm{w}$ gabapentin gel significantly alleviated both the mechanical and cold allodynia as well as mechanical and thermal hyperalgesia. The topical gabapentin $10 \% \mathrm{w} / \mathrm{w}$ gel preparation tested in this study possessed minimal adverse effects 
compared to those associated with systemic gabapentin, which may be ascribed to the lower systemic concentration of active ingredient delivered by the topical formulation. Therefore, the gel formulation of gabapentin as a topical agent could be a better alternative for the clinical management of some neuropathic pain syndromes.

\section{Conclusions:}

The present study offered a relatively broad pharmacological validation of topical gabapentin gel in the chronic constriction injury model of neuropathic pain. Both gabapentin gel and systemic gabapentin induced comparable profiles of activity, as they both evoked significant antihyperalgesic and anti-allodynic properties. Topical gabapentin gel offers the advantage that it is devoid of side effects associated with the systemic use of gabapentin. The topical gel formulation of gabapentin warrants further evaluation in other neuropathic pain models. However, it must be kept in mind that there are differences between animal models in terms of the magnitude of each pain component. Additionally, there is the dilemma of translation from animal pain models into effective pain therapy so the importance of patient studies cannot be overemphasized.

\section{Acknowledgements:}

We gratefully acknowledge the supply of gabapentin gel $10 \% \mathrm{w} / \mathrm{w}$ and control gel (base minus the API) by SMPU (Cardiff, UK) under their MS. We also acknowledge the gift of gabapentin by Lowitt Pharmaceuticals (Pvt.) Ltd., Peshawar, Pakistan.

\section{Author contributions:}

F.S guided the research group as supervisor in planning and conducting experiments. M.S performed the experiments and carried out statistical analysis. He also prepared the final version 
of the manuscript. N.A, G.A, S.A and K.F provided their help throughout the study. RDES guided the research group and interpreted the results as well as critically revised the manuscript for important intellectual content.

\section{References:}

Ali, G., Subhan, F., Abbas, M., Zeb, J., Shahid, M., and Sewell, R.D. (2015). A streptozotocininduced diabetic neuropathic pain model for static or dynamic mechanical allodynia and vulvodynia: Validation using topical and systemic gabapentin. Naunyn Schmiedebergs Arch Pharmacol 388, 1129-1140.

Attal, N. (2001). Pharmacologic treatment of neuropathic pain. Acta Neurol Belg 101, 53-64.

Austin, P.J., Wu, A., and Moalem-Taylor, G. (2012). Chronic constriction of the sciatic nerve and pain hypersensitivity testing in rats. Journal of visualized experiments: JoVE 61, e3393.

Bennett, G.J., Chung, J.M., and Seltzer, Z.e. (2003). Models of neuropathic pain in the rat. Curr Protoc Pharmacol 9.14.

Bennett, G.J., and Xie, Y.-K. (1988). A peripheral mononeuropathy in rat that produces disorders of pain sensation like those seen in man. Pain 33, 87-107.

Bennett, M.I., and Simpson, K.H. (2004). Gabapentin in the treatment of neuropathic pain. Palliat Med 18, 5-11.

Bouhassira, D., Wilhelm, S., Schacht, A., Perrot, S., Kosek, E., Cruccu, G., Freynhagen, R., Tesfaye, S., Lledó, A., and Choy, E. (2014). Neuropathic pain phenotyping as a predictor of treatment response in painful diabetic neuropathy: data from the randomized, double-blind, COMBO-DN study. Pain 155, 2171-2179.

Campbell, J.N., and Meyer, R.A. (2006). Mechanisms of neuropathic pain. Neuron 52, 77-92.

Carter, R.J., Morton, J., and Dunnett, S.B. (2001). Motor coordination and balance in rodents. Curr Protoc Neurosci Unit 8.12.

Chaplan, S., Bach, F., Pogrel, J., Chung, J., and Yaksh, T. (1994). Quantitative assessment of tactile allodynia in the rat paw. $J$ Neurosci Methods 53, 55-63.

Chen, L., Chen, W., Qian, X., Fang, Y., and Zhu, N. (2014). Liquiritigenin alleviates mechanical and cold hyperalgesia in a rat neuropathic pain model. Sci Rep 4, 5676. 
Costigan, M., Scholz, J., and Woolf, C.J. (2008). Neuropathic pain: A maladaptive response of the nervous system to damage. Annu Rev Neurosci 32, 1-32.

De Vry, J., Kuhl, E., Franken-Kunkel, P., and Eckel, G. (2004). Pharmacological characterization of the chronic constriction injury model of neuropathic pain. Eur J Pharmacol 491, 137-148.

Decosterd, I., and Woolf, C.J. (2000). Spared nerve injury: an animal model of persistent peripheral neuropathic pain. Pain 87, 149-158.

Field, M., Oles, R., Lewis, A., McCleary, S., Hughes, J., and Singh, L. (1997). Gabapentin (neurontin) and S-(+)-3-isobutylgaba represent a novel class of selective antihyperalgesic agents. Br J Pharmacol 121, 1513-1522.

Gilron, I., and Flatters, S.J. (2006). Gabapentin and pregabalin for the treatment of neuropathic pain: A review of laboratory and clinical evidence. Pain Res Manag 11, 16A-29A.

Harden, R.N. (1999). Gabapentin: a new tool in the treatment of neuropathic pain. Acta Neurol Scand 100, 43-47.

Hiom, S., Khot, S., Mogford, S., Hart, C., Patel, G., Roberts, G., Martin, C., and Newcombe, R. (2015a). Topical delivery of gabapentin (Gaba Gel $\left.{ }^{\mathrm{TM}}\right)$ for neuropathic pain: A' proof of concept' study. Int J Pharm Pract 23, 46.

Hiom, S., Patel, G., Newcombe, R., and Khot, S. (2015b). Severe postherpetic neuralgia and other neuropathic pain syndromes alleviated by topical gabapentin. Br J Dermatol 173, 300-302.

IASP (1994). Pain terms: a current list with definitions and notes on usage. (Classification of Chronic Pain, 2nd edition, IASP Task Force on Taxonomy, eds H. Merskey, H., Bogduk, N. IASP Press, Washington DC), pp. 209-214. updated 2014. http://www.iasp-pain.org.

Jensen, T.S., and Finnerup, N.B. (2014). Allodynia and hyperalgesia in neuropathic pain: clinical manifestations and mechanisms. Lancet Neurol 13, 924-935.

Mao-Ying, Q.-L., Kavelaars, A., Krukowski, K., Huo, X.-J., Zhou, W., Price, T.J., Cleeland, C., and Heijnen, C.J. (2014). The anti-diabetic drug metformin protects against chemotherapyinduced peripheral neuropathy in a mouse model. PLOS ONE 9, e100701.

Marchettini, P., Wilhelm, S., Petto, H., Tesfaye, S., Tölle, T., Bouhassira, D., Freynhagen, R., Cruccu, G., Lledó, A., and Choy, E. (2016). Are there different predictors of analgesic response between antidepressants and anticonvulsants in painful diabetic neuropathy? Eur J Pain 20, 472482. 
McLean, M.J., Morrell, M.J., Willmore, L.J., Privitera, M.D., Faught, R.E., Holmes, G.L., Magnus-Miller, L., Bernstein, P., and Rose-Legatt, A. (1999). Safety and tolerability of gabapentin as adjunctive therapy in a large, multicenter study. Epilepsia 40, 965-972.

Mendell, J.R., and Sahenk, Z. (2003). Painful sensory neuropathy. N Engl J Med 348, 12431255.

Moulin, D., Boulanger, A., Clark, A., Clarke, H., Dao, T., Finley, G., Furlan, A., Gilron, I., Gordon, A., and Morley-Forster, P. (2014). Pharmacological management of chronic neuropathic pain: revised consensus statement from the Canadian Pain Society. Pain Res Manag 19, 328-335.

Moulin, D., Clark, A., Gilron, I., Ware, M., Watson, C., Sessle, B., Coderre, T., Morley-Forster, P., Stinson, J., and Boulanger, A. (2007). Pharmacological management of chronic neuropathic pain-consensus statement and guidelines from the Canadian Pain Society. Pain Res Manag 12, 13.

Nakazato-Imasato, E., Tanimoto-Mori, S., and Kurebayashi, Y. (2009). Effect of mexiletine on dynamic allodynia induced by chronic constriction injury of the sciatic nerve in rats. $J$ Vet Med Sci 71, 991-994.

NICE (2013). Neuropathic pain: the pharmacological management of neuropathic pain in adults in non-specialist settings. https://wwwniceorguk/guidance/CG173/chapter/1-Recommendations.

Rose, M., and Kam, P. (2002). Gabapentin: pharmacology and its use in pain management. Anaesthesia 57, 451-462.

Rowbotham, M., Harden, N., Stacey, B., Bernstein, P., Magnus-Miller, L., and Group, G.P.N.S. (1998). Gabapentin for the treatment of postherpetic neuralgia: a randomized controlled trial. JAMA 280, 1837-1842.

Sawynok, J. (2014). Topical analgesics for neuropathic pain: preclinical exploration, clinical validation, future development. Eur J Pain 18, 465-481.

Stanos, S.P. (2007). Topical agents for the management of musculoskeletal pain. J Pain Symptom Manage 33, 342-355.

Subhan, F., Karim, N., Gilani, A.H., and Sewell, R.D. (2010). Terpenoid content of Valeriana wallichii extracts and antidepressant-like response profiles. Phytotherapy Research 24, 686-691.

Zur, E. (2014). Topical treatment of neuropathic pain using compounded medications. ClinJPain 30, 73-91. 


\section{FIGURES}

(A) Static allodynia

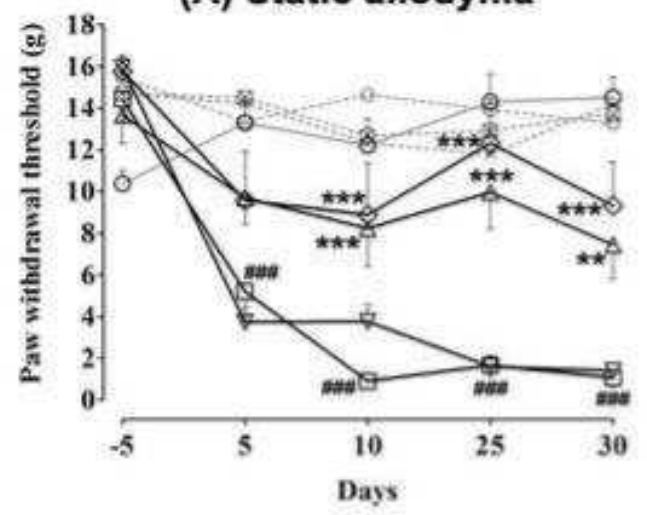

(C) Heat hyperalgesia (PWL)

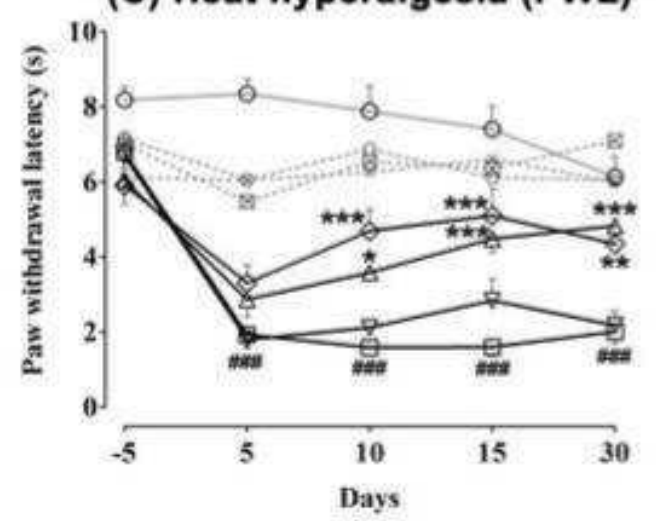

(B) Dynamic allodynia

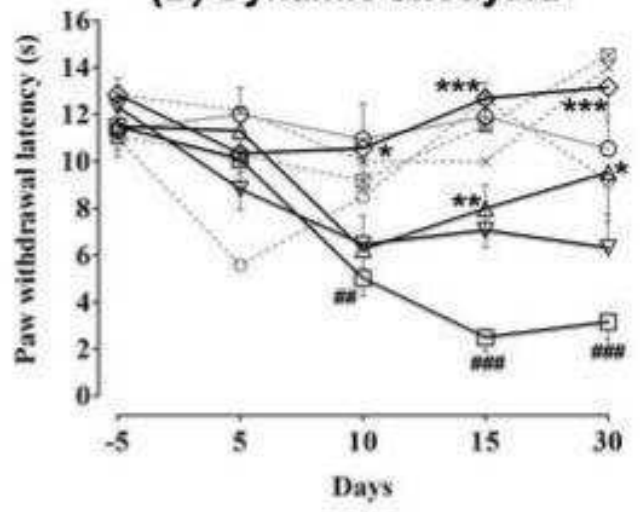

(D) Heat hyperalgesia (PWD)

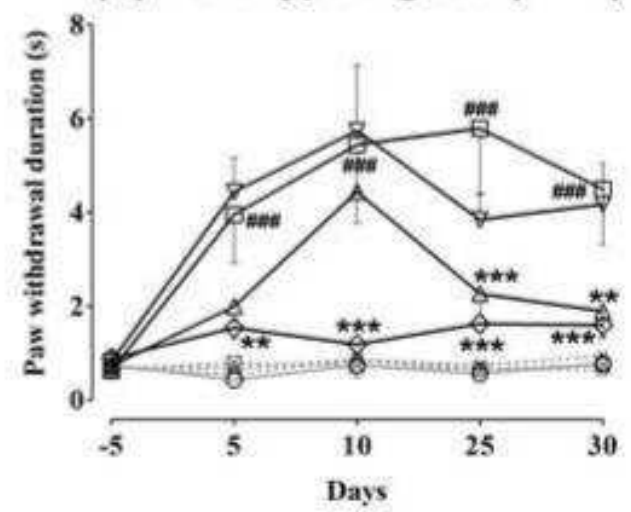

$\diamond \mathrm{CCI}+\mathrm{GP}-\mathrm{IP}$

- GG

Figure 1: Effect of topical ipsilateral paw 10\% gabapentin gel (GG), ipsilateral paw control gel (CG) and systemic gabapentin (75 mg/kg i.p., GP) on chronic constriction injury (CCI) induced (A): static allodynia [diminished von Frey filament threshold pressure; PWT (g)], (B): dynamic allodynia [diminshed paw withdrawal latency to light brushing; PWL (s)] and heat hyperalgesia [shortened paw withdrawal latency (PWL) (C), and increased paw withdrawal duration (PWD) (D) to heat stimulus]. Values expressed as mean \pm SEM. ${ }^{\#} P<0.01,{ }^{\# \#} P<0.001$ compared to sham operated animals, $* P<0.05,{ }^{* *} P<0.01, * * * P<0.001$ compared to CCI operated untreated animals, two-way repeated measures ANOVA followed by post hoc Bonferroni analysis. $n=6$ rats per group. 


\section{(A) Mechano-hyperalgesia}

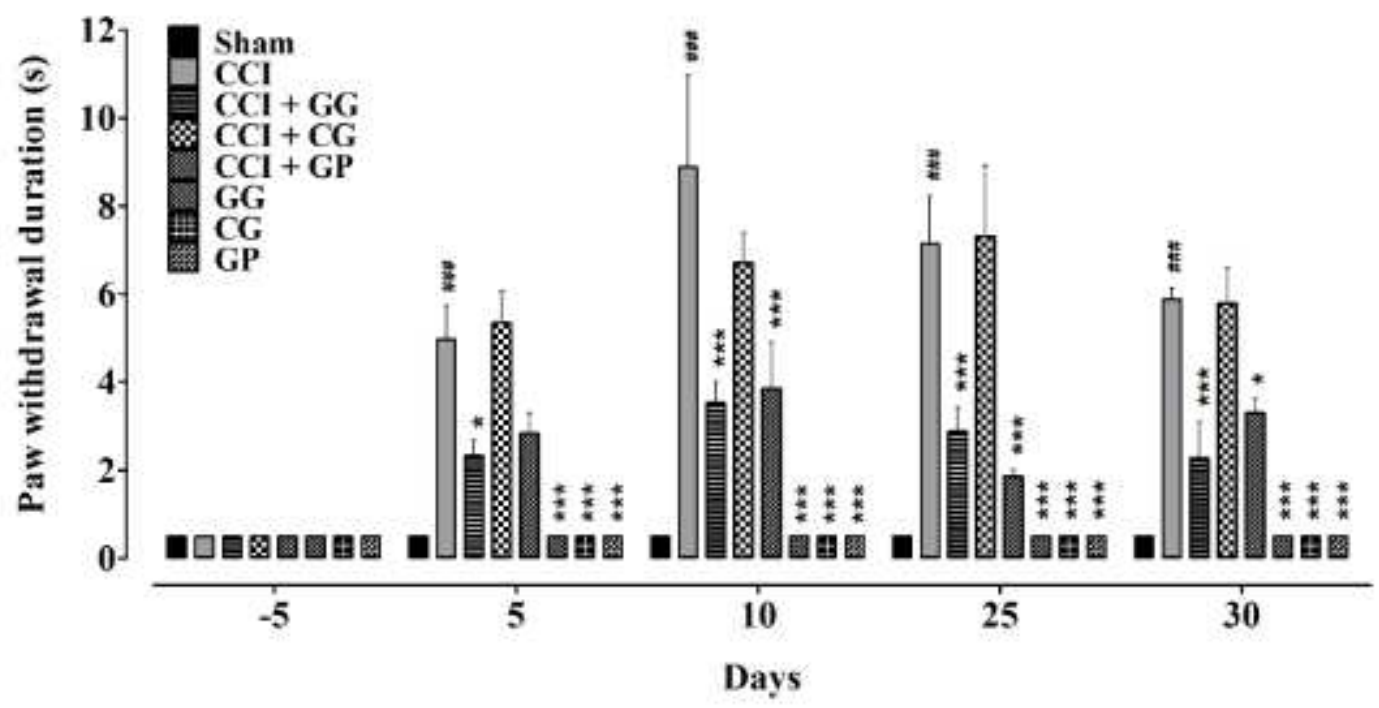

(B) Cold allodynia

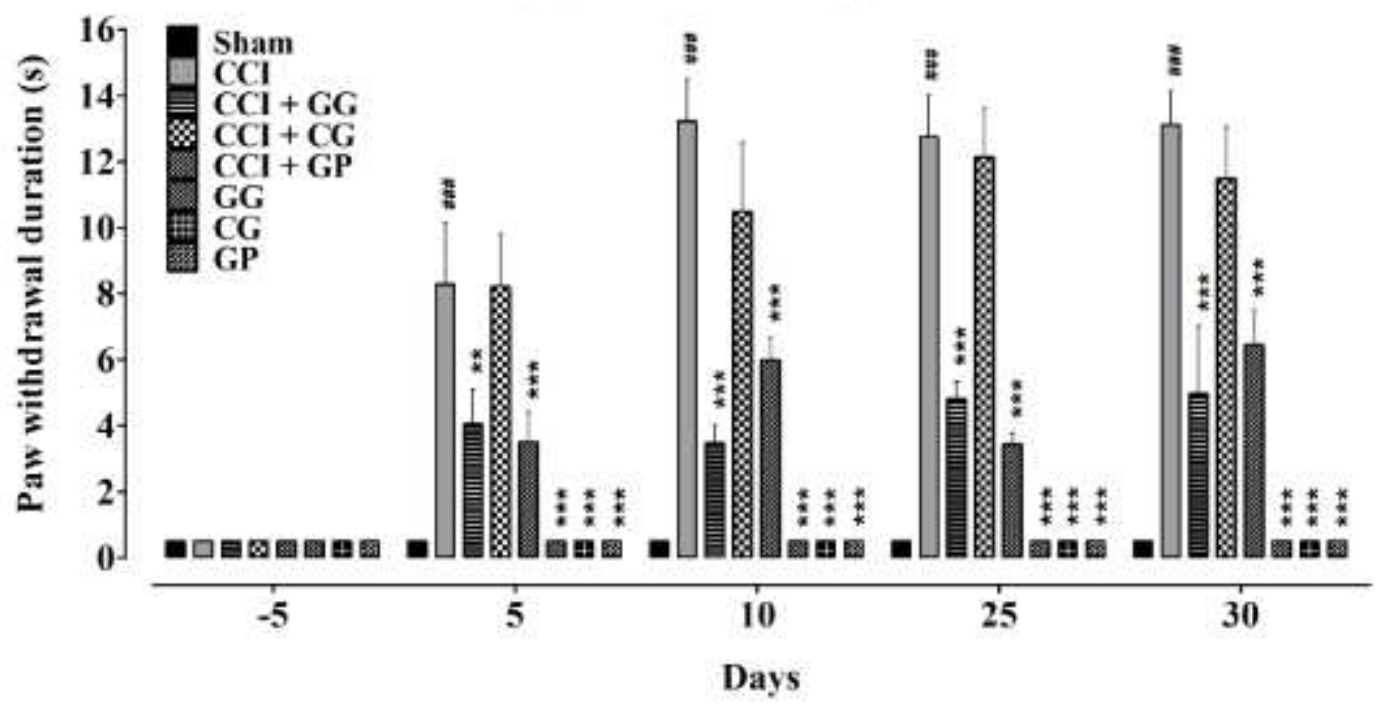

Figure 2: Effect of topical ipsilateral paw 10\% gabapentin gel (GG), ipsilateral paw control gel (CG) and systemic gabapentin (GP) on chronic constriction injury (CCI) induced (A): mechanical hyperalgesia [increased paw withdrawal duration (PWD) to pin prick] and (B): cold allodynia [increased paw withdrawal duration (PWD) to a drop of acetone]. Values expressed as mean \pm SEM. ${ }^{\# \# \#} P<0.001$ compared to sham operated animals, $* P<0.05, * * P<0.01$, ***P< 0.001 compared to CCI operated untreated animals, two-way repeated measures ANOVA followed by post hoc Bonferroni analysis. $n=6$ rats per group. 
(A) Static allodynia

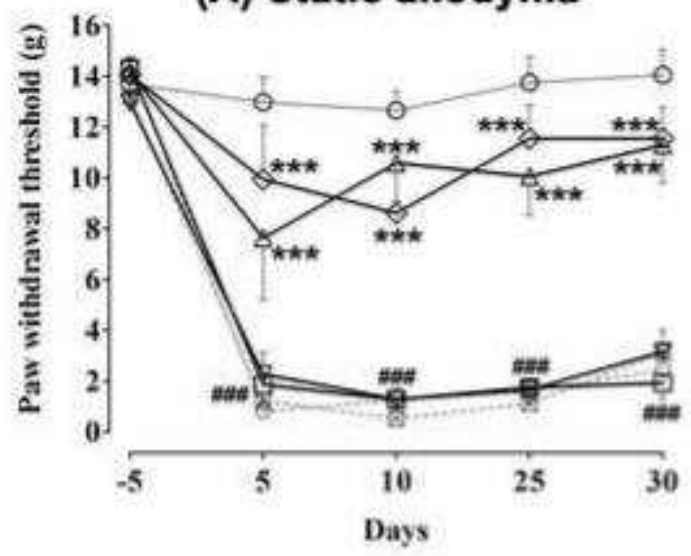

(C) Heat hyperalgesia (PWL)

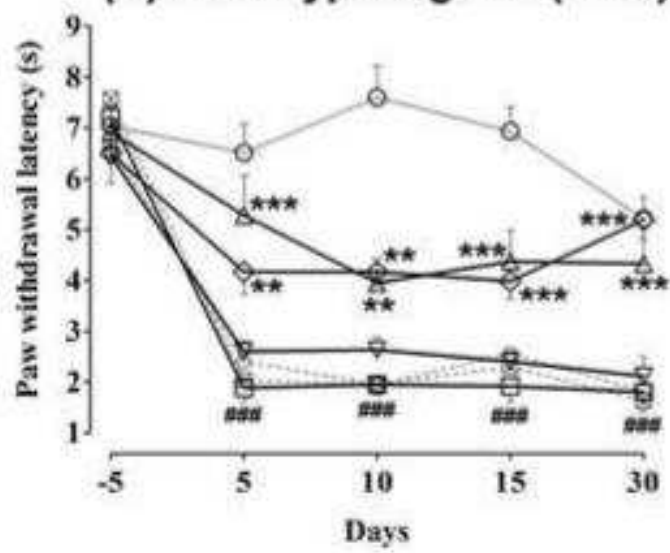

- Sham
$\square \mathrm{CCI}$ $\triangle \mathrm{CCI}+\mathbf{G G}$

$\nabla \mathrm{CCI}+\mathrm{CG}$

\& $\mathbf{C C I}+\mathbf{C G}-$ Contra
(B) Dynamic allodynia

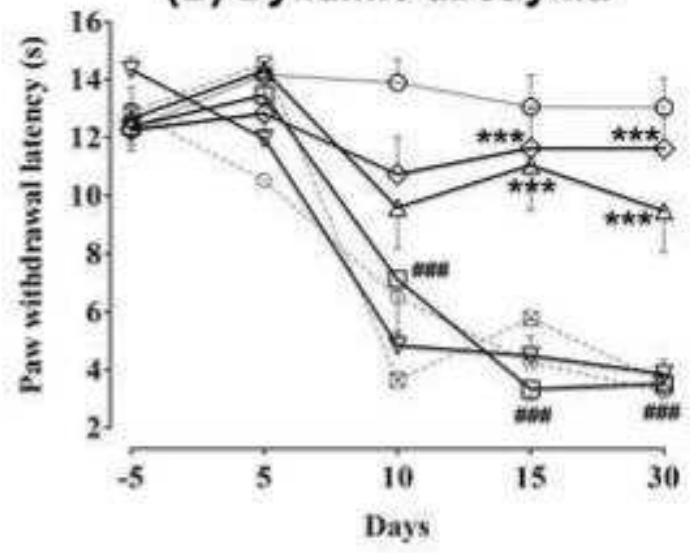

(D) Heat hyperalgesia (PWD)

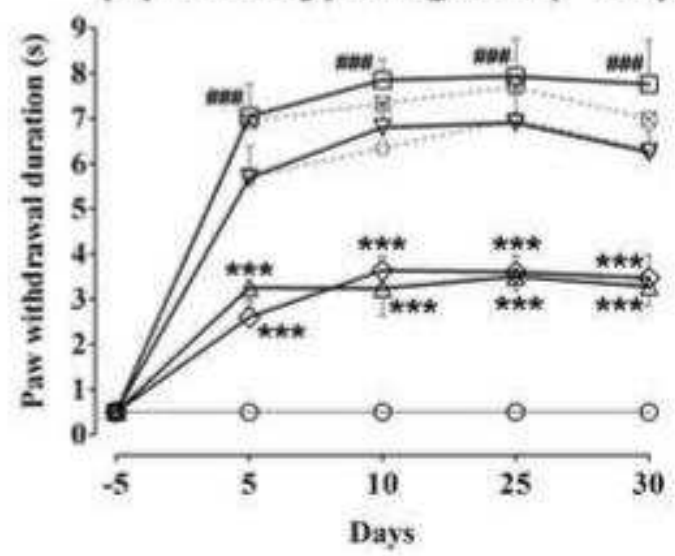

$\diamond \mathrm{CCI}+\mathrm{GP}-\mathrm{IP}$

CCl + GG-Contra

Figure 3: Effect of topical contralateral paw 10\% gabapentin gel (GG), contralateral paw control gel (CG) and systemic gabapentin (GP) on chronic constriction injury (CCI) induced (A): static allodynia [diminished von Frey filament threshold pressure; PWT (g)], (B): dynamic allodynia [diminshed paw withdrawal latency to light brushing; PWL (s)] and heat hyperalgesia [shortened paw withdrawal latency (PWL) (C), and increased paw withdrawal duration (PWD) (D) to heat stimulus]. Values expressed as mean \pm SEM. ${ }^{\# \#} P<0.001$ compared to sham operated animals, $* * P<0.01, * * * P<0.001$ compared to CCI operated untreated animals, two-way repeated measures ANOVA followed by post hoc Bonferroni analysis. $n=6$ rats per group. 
(A) Mechano-hyperalgesia

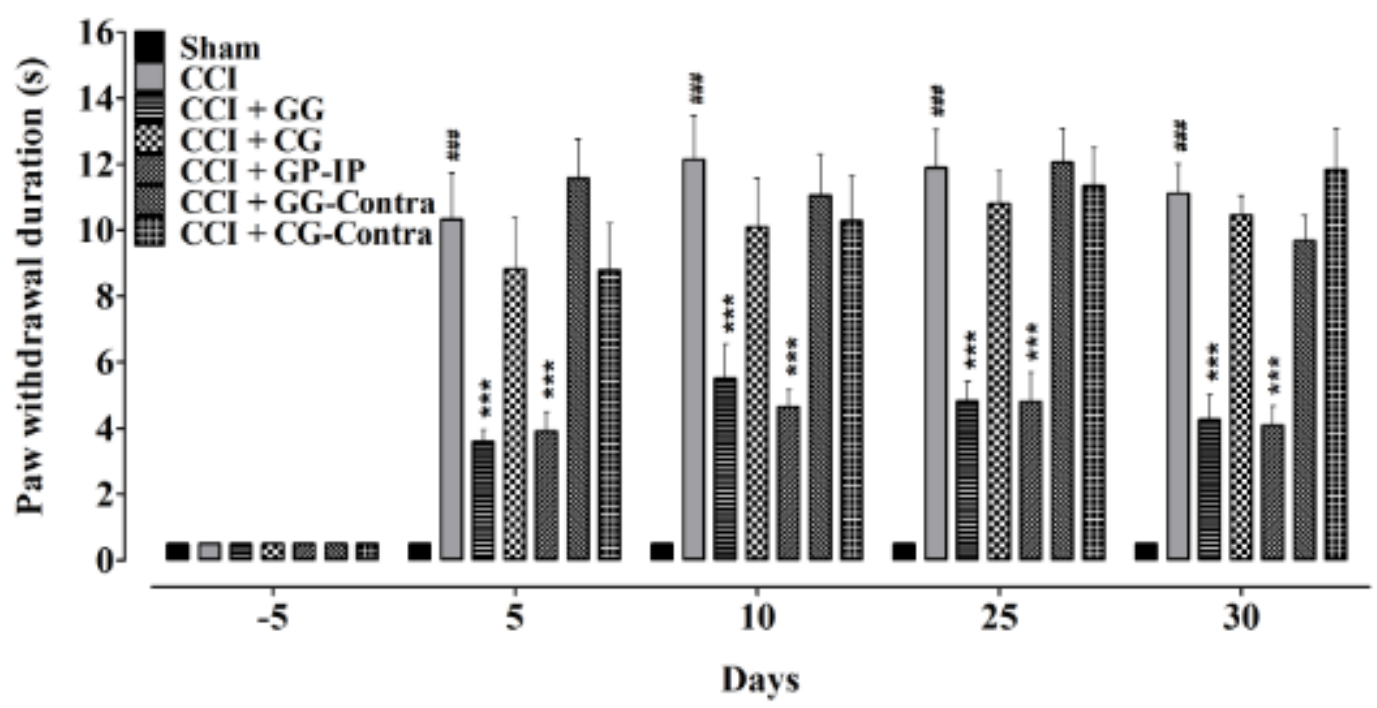

(B) Cold allodynia

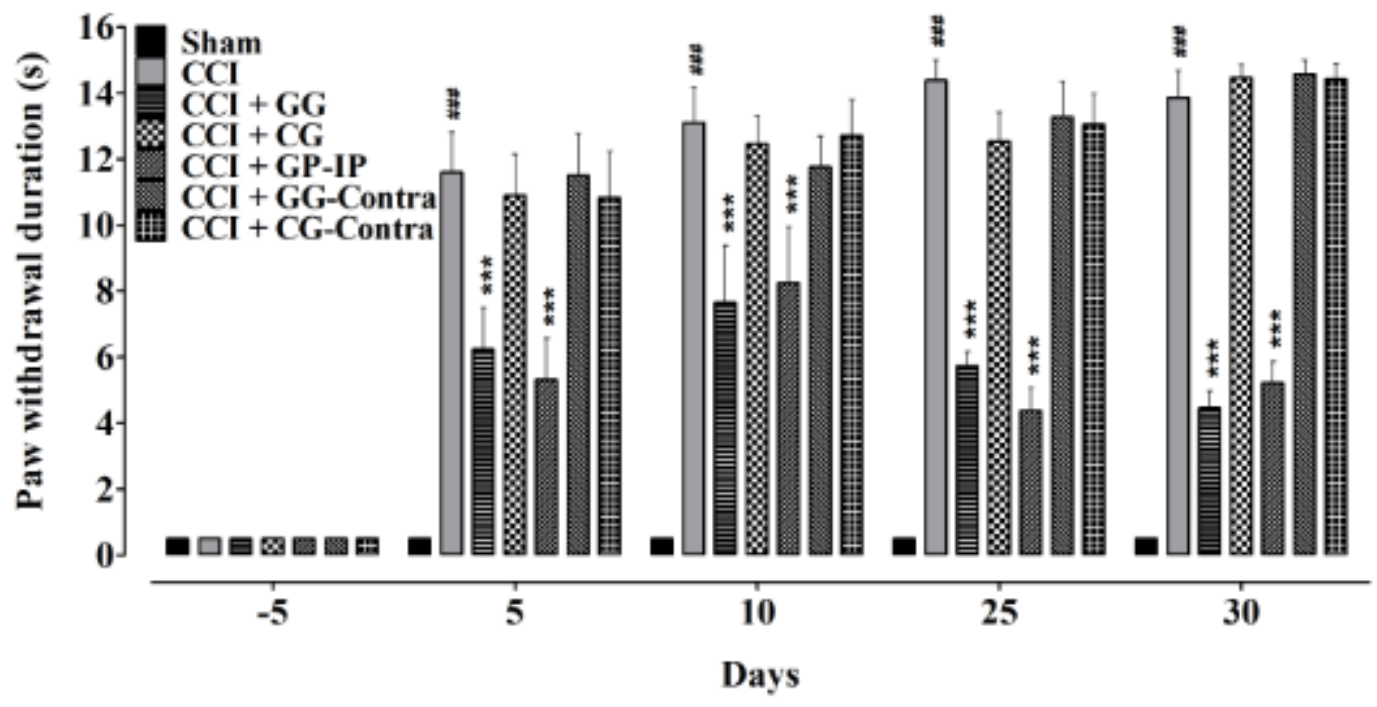

Figure 4: Effect of topical contralateral paw 10\% gabapentin gel (GG), contralateral paw control gel (CG) and systemic gabapentin (GP) on chronic constriction injury (CCI) induced (A): mechanical hyperalgesia [increased paw withdrawal duration (PWD) to pin prick] and (B): cold allodynia [increased paw withdrawal duration (PWD) to a drop of acetone]. Values expressed as mean \pm SEM. ${ }^{\# \#} P<0.001$ compared to sham operated animals, ${ }^{* * *} P<0.001$ compared to CCI operated untreated animals, two-way repeated measures ANOVA followed by post hoc Bonferroni analysis. $n=6$ rats per group. 


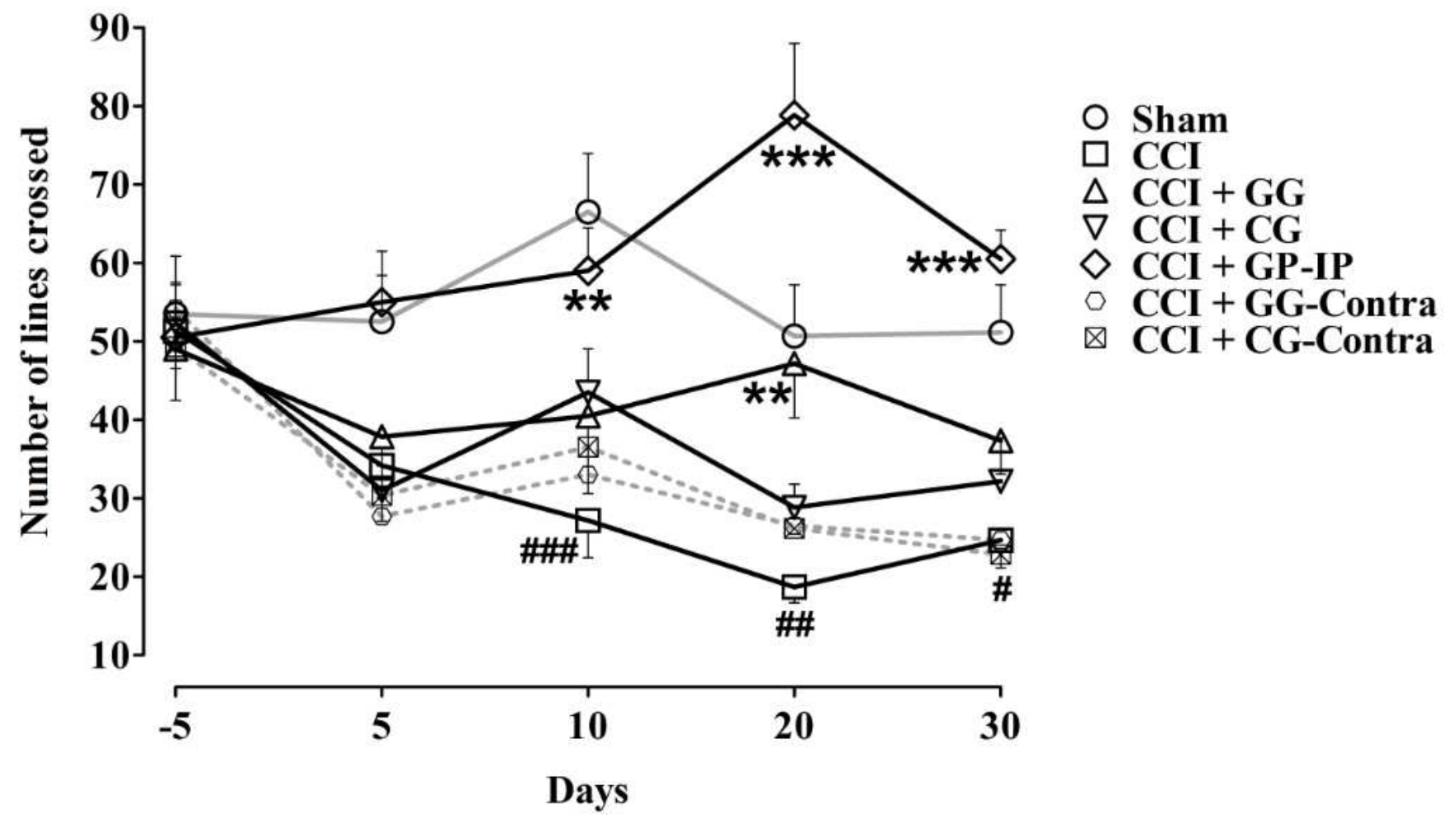

Figure 5: Effect of topical ipsilateral or contralateral paw 10\% gabapentin gel (GG), ipsilateral or contralateral paw control gel (CG) and systemic gabapentin (GP) on the general locomotor activity in chronic constriction injury (CCI) animals expressed as the total number of lines crossed in a behavioral arena in $20 \mathrm{~min}, 1 \mathrm{~h}$ post treatment. Values expressed as mean \pm SEM. ${ }^{\#} P$

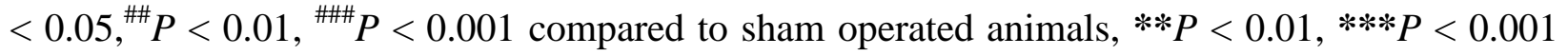
compared to CCI operated untreated animals, two-way repeated measures ANOVA followed by post hoc Bonferroni analysis. $n=6$ rats per group. 


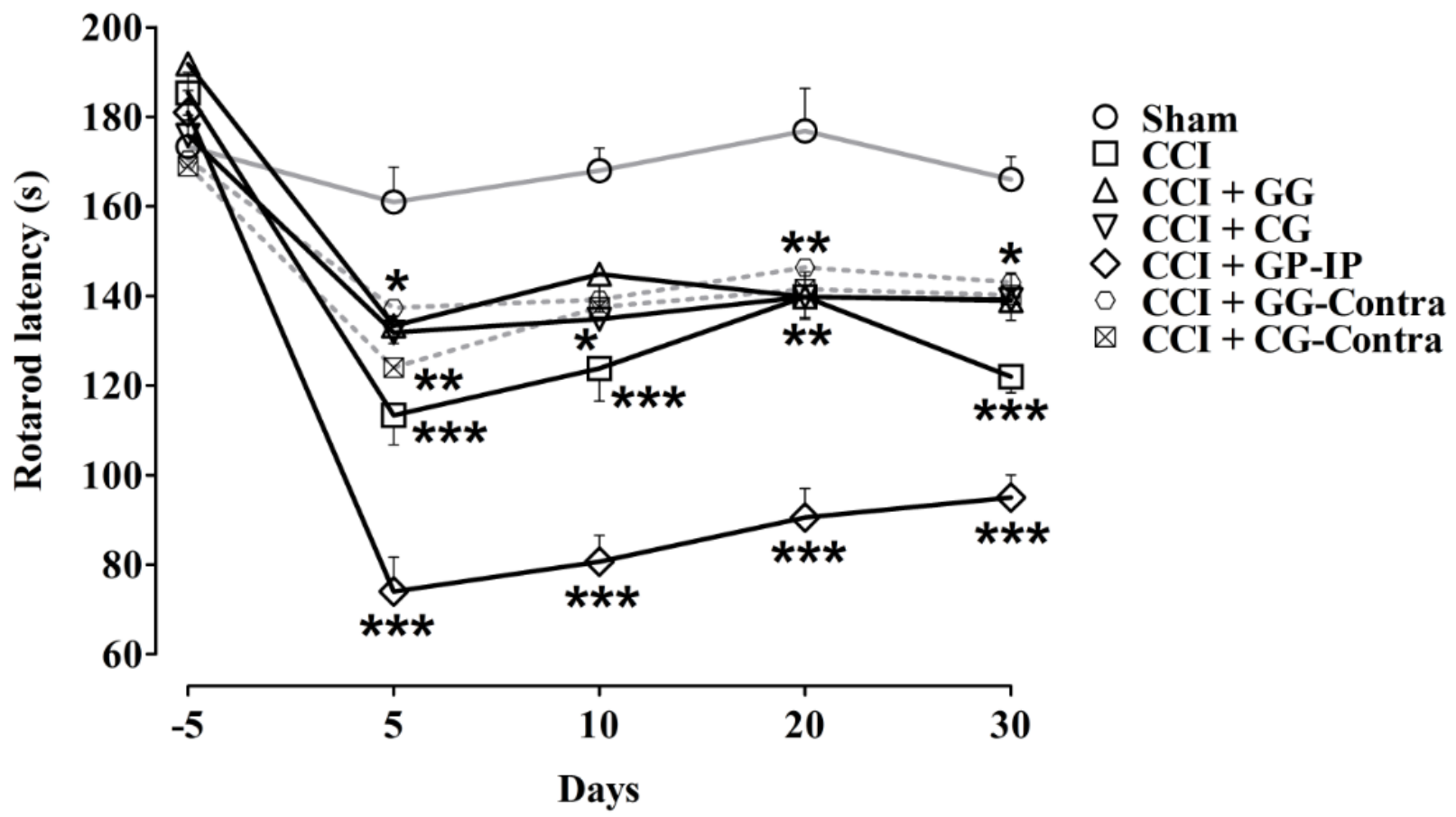

Figure 6: Effect of topical ipsilateral or contralateral paw 10\% gabapentin gel (GG), ipsilateral or contralateral paw control gel (CG) and systemic gabapentin (GP) on the rotarod performance in chronic constriction injury (CCI) animals expressed as the drop-off latency, $1 \mathrm{~h}$ post treatment. Values expressed as mean \pm SEM. ${ }^{*} P<0.05$, ${ }^{* *} P<0.01, * * * P<0.001$ compared to sham operated animals, two-way repeated measures ANOVA followed by post hoc Bonferroni analysis. $n=6$ rats per group. 


\section{(A) Stride length}

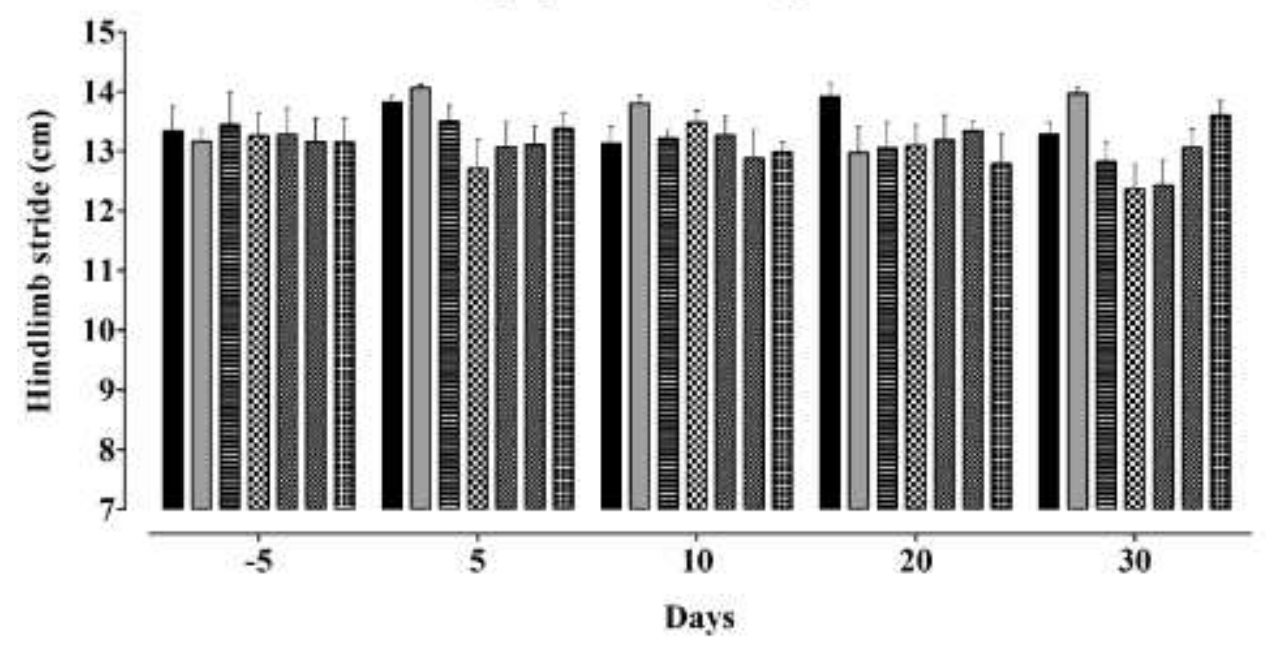

(B) Forepaw and hindpaw overlap

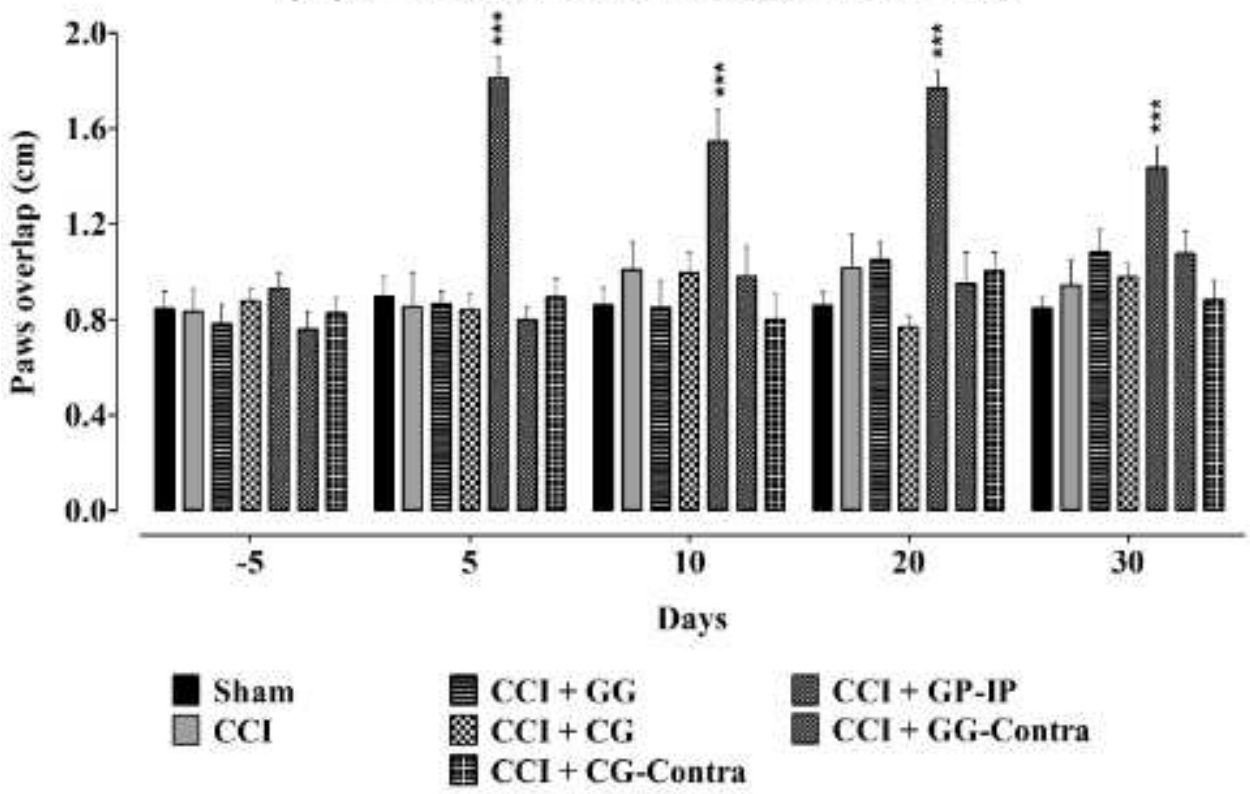

Figure 7: Effect of topical ipsilateral or contralateral paw 10\% gabapentin gel (GG), ipsilateral or contralateral paw control gel (CG) and systemic gabapentin (GP) on the footprint analysis in chronic constriction injury (CCI) animals expressed as the distance of forward movement between each stride (stride length) (A), and overlap between forepaw and hindpaw placement (paw overlap) (B), $1 \mathrm{~h}$ post treatment. Values expressed as mean \pm SEM. ${ }^{*} * * P<0.001$ compared to sham operated animals, two-way repeated measures ANOVA followed by post hoc Bonferroni analysis. $n=6$ rats per group. 